\title{
TRATAMIENTO DE LA FORMACIÓN DE PALABRAS EN GRAMÁTICAS DEL ESPAÑOL DEL SIGLO XIX
}

\author{
Marta TORRES Martínez \\ Universidad de Jaén \\ matorma@ujaen.es
}

\section{Resumen}

Este artículo se centra en analizar el tratamiento de la formación de palabras en una selección de gramáticas del español publicadas a lo largo del siglo XIX, adscritas tanto a la corriente académica como a la no académica.

La formación de palabras ha gozado de escasa atención en la tradición gramatical española, de tal modo que no se trata de manera autónoma -a modo de apartado o capítulohasta el primer cuarto del siglo XX, tanto en el caso de la RAE como en el de los autores ajenos a la esta institución. Habitualmente las cuestiones sobre formación de palabras se han ubicado en los capítulos destinados a explicar las distintas clases de palabras o partes de la oración -en concreto, en los relativos al sustantivo, adjetivo y verbo, categorías léxicas que, junto con el adverbio, presentan posibilidades derivativas-, debido a la influencia de la tradición gramatical clásica.

En nuestro trabajo analizamos la presencia de las cuestiones concernientes a la formación de palabras en los tratados decimonónicos seleccionados a fin de comprobar si, además de la influencia grecolatina, hallamos propuestas novedosas en relación con la información morfológica que nos ocupa.

PALABRAS CLAVE: Formación de palabras (composición, derivación y parasíntesis), tradición gramatical, siglo XIX.

\begin{abstract}
This article focuses on the analysis of the treatment of word formation in a selection of grammar books of both academic and non-academic trends, published in the XIXth century.

Such little attention has been paid to word formation in traditional Spanish grammars that it has not been treated autonomously -in separate sections or chapters- until the first quarter of the XXth century. This is true of both the RAE (Real Academia Española) and of authors outside this scholarly institution. Matters related to word formation have normally been placed in chapters dedicated to the explanation of different types of words or parts of a sentence -such as nouns, adjectives or verbs, lexical categories with derivational possibilities together with adverbs-, due to the influence of classical grammatical tradition.

The present study analyses the presence of issues concerned with word formation in selected nineteenth century treatises in order to ascertain whether, as well as noticing their Greek and Latin influence, we find new proposals as regards the morphological information in which we are interested.
\end{abstract}

KEY WORDS: Word formation (compounding, derivation and parasynthesis), grammatical tradition, XIXth century.

\section{Introducción}

El objetivo del presente trabajo consiste en analizar, valorar e interpretar el tratamiento que otorgan algunas gramáticas del español publicadas a lo largo del siglo XIX a la formación de palabras. 
En concreto, nuestro interés radica en conocer tres cuestiones fundamentales: (i) si las gramáticas decimonónicas seleccionadas incluyen aspectos sobre formación de palabras, (ii) en qué lugar o en qué parte de estas obras se describe tal materia y (iii) qué tipo de procedimientos se contemplan (composición, derivación y parasíntesis).

El corpus analizado incluye textos gramaticales adscritos a dos tradiciones distintas: por un lado, nos centramos en algunas de las gramáticas concebidas en el seno de la Real Academia Española (GRAEs 1854, 1858, 1870, 1874 y 1880) y, por otro, atendemos a gramáticas proyectadas por autores ajenos a la Corporación (Salvá, 1830; Bello, 1847; Herrainz, 1885; Salleras, 1876 y Blanco, 1896).

En definitiva, a fin de esbozar el panorama que ofrece la tradición gramatical española decimonónica, creemos interesante comparar el tratamiento que recibe la formación de palabras en textos académicos y no académicos. Este cotejo nos proporcionará un conocimiento más profundo de la evolución de la información morfológica en esta etapa concreta de la historia de la gramática española.

\section{La formación de palabras en la tradición gramatical del español}

Basta con hacernos eco de las siguientes palabras de Montero (1998: 294) para entender que la formación de palabras ha gozado de escasa atención en la historia de la gramática del español:

La carencia de estudios sobre la formación de palabras puede tener su origen en el tratamiento que han dado los gramáticos a este tema. Si una gramática se puede considerar como manual de primera mano a la hora de abordar un análisis lingüístico y se observa que en estas obras la formación de palabras no ha merecido interés, podrá entenderse que los trabajos específicos hayan sido también muy escasos.

En la línea de Montero (1998), otros autores como Sala (1989: 268), García Platero (1998: 71) y Serrado-Dolader (2001: 407) señalan que, de hecho, la formación de palabras no se trata de manera autónoma hasta el primer cuarto del siglo XX, cuando aparece el Tratado de formación de palabras en la lengua española. La derivación y la composición (1920) de José Alemany Bolufer ${ }^{1}$.

No obstante, aunque no integradas en un capítulo destinado exclusivamente a tal materia, algunas cuestiones sobre formación de palabras son

1 Además de ser publicado en la imprenta de Victoriano Suárez en el año 1920, este texto vio la luz anteriormente en varios fascículos del Boletín de la Real Academia Española publicados entre 1917 y 1919. 
explicadas ya por los gramáticos grecolatinos², según apunta González Ruiz (1994: 271):

La única información que la mayor parte de los gramáticos latinos dieron sobre formación de palabras (procedimientos de derivación y de composición) estaba situada en los "accidentes" de la figura y de la especie, y más concretamente en la aplicación de estos accidentes al nombre y al verbo, ya que en estas dos partes de la oración quedaban incluidas las tres categorías léxicas con posibilidades derivativas (sustantivo, adjetivo y verbo).

En efecto, tanto Ramajo (1987: 78) como González Ruiz (1994: 273), entre otros autores, comentan que la especie y la figura ${ }^{3}$-junto al género, número y caso- formaban parte de los accidentes del nombre establecidos por Dionisio de Tracia (s. I a. C.). De una parte, el gramático griego, en virtud de la especie, dividía el nombre en primitivo y derivado y, a su vez, dentro de este último se consideraban los patronímicos, posesivos, verbales, comparativos, superlativos o diminutivos. De otra parte, mediante la figura Dionisio de Tracia distinguía los nombres simples de los compuestos (cfr. Ramajo, 1987: 94 y González Ruiz, 1994: 273) ${ }^{4}$.

Ya en la tradición gramatical española González Ruiz (1994: 273) comprueba que Nebrija y Correas se ocupan de la especie y la figura al tratar el nombre y el verbo ${ }^{5}$. A propósito de la descripción llevada a cabo por estos gramáticos, González Ruiz (1994: 273) señala que "ellos son los únicos que dan información algo detallada sobre los procedimientos de derivación y composición en español, sobre todo en relación con sufijos derivativos, estudiados en la especie del nombre".

2 Montero (1998: 295, nota 4) recuerda que "pueden rastrearse opiniones o explicaciones parciales sobre la formación de palabras ya desde la primera gramática de Panini". En concreto, la autora añade que "se trata de la distinción entre tatpurusha (tipo de compuesto que se aproxima al grupo libre de dos sustantivos [...] del tipo español hojalata)", bahuvrihi (compuesto que resulta de la transformación de un nombre compuesto en un adjetivo [...], como carilindo) y dvandva (tipo de compuestos en el que los dos miembros [...] mantienen una relación coordinativa, sin que exista la primacía de uno de los dos términos sobre el otro, son formas como casatienda)".

3 Los términos griegos referidos a la especie y la figura fueron eîdos y schêma, respectivamente.

4 En cuanto a la figura, al estudiar las gramáticas de la lengua castellana desde Nebrija a Correas, Ramajo (1987: 95) afirma que "curiosamente, los gramáticos no han prestado atención, salvo excepciones, a este accidente. Han preferido tratar las palabras más desde un punto de vista lexicográfico, quiero decir, atendiendo a su significado, que desde un punto de vista morfológico. En esta última faceta se limitan, por lo general, al estudio del género, número y caso, que para ellos, eran los auténticos accidentes del nombre".

5 Por su parte, Montero (1998: 295) indica que "Nebrija también es ya consciente de las diferencias entre composición y derivación y así las expone en su obra aplicadas a las distintas clases de palabras". 
Igualmente, al estudiar el tratamiento de la formación de palabras en gramáticas del español publicadas desde 1492 hasta 1771, Suárez (1999: 627628) afirma lo siguiente:

La generalidad de las gramáticas muestra un interés muy superior por los rasgos inherentes a ciertas clases de palabras (género y número en el nombre y tiempo, modo y voz en el verbo) que por los procedimientos de formación o, si se quiere, visto desde el ángulo opuesto, que por los tipos de palabras según la clase de base sobre la que se han formado 6 .

Gómez Asencio (1981: 348) apunta el motivo por el que la formación de palabras no alcanza peso suficiente como para integrar un capítulo en las gramáticas al uso o como para ser tratada en un texto independiente. Este autor señala que la palabra es la unidad fundamental y básica del estudio lingüístico realizado en España entre 1771 y 1847, periodo analizado en su investigación. En concreto, Gómez Asencio (1981: 348) indica que los gramáticos someten la palabra a tres tipos de análisis:

(i) a un análisis segmental y taxonómico que trata de descomponer las palabras en elementos menores (raíz; prefijos, sufijos; formas de género y número; de tiempo, modo...), cuyo valor morfosintáctico y aportación semántica es en general bien percibida y puesta de manifiesto; (ii) a un análisis de la actuación sintáctica de las palabras en el que, salvo en muy raras excepciones, no se parte tanto de la descomposición del sintagma (oración, proposición, frase...) concebido como un todo unitario, cuanto de la observación de la palabra -unidad tomada (casi) siempre como referencia- en el sintagma; aquí se estudian fundamentalmente los fenómenos de concordancia y régimen, y en grado mínimo las clases de oraciones; (iii) a un tercer análisis (semántico o sintáctico, intraoracional o extraoracional) por el que se pretende agrupar todos los vocablos existentes en una lengua o todas las voces posibles en el lenguaje -según las intenciones, particulares o generales, del autor- en una serie de paradigmas, a los que se conoce en la época con los nombres de partes de la oración o (clases de) palabras.

Para los gramáticos de la época el tercer análisis era la base de los otros dos, de hecho, según afirma Gómez Asencio (1981: 348), "la morfología de la palabra (i) y la sintaxis de la palabra (ii), se montan después de éste, en torno a éste y utilizando las unidades obtenidas y definidas en éste".

Ello explica que, según señala este especialista, sea difícil hallar un tratado sobre la composición de la palabra, pues "a cambio encontraremos frecuentes referencias a los nombres compuestos, los verbos compuestos...".

6 No obstante, como venimos observando, Suárez (1999) comprueba que los tratados gramaticales de Nebrija y Correas así como la GRAE (1771) contienen aspectos relacionados con los procesos de formación al describir ciertas clases de palabras. 


\section{La formación de palabras en gramáticas del siglo XIX}

Según hemos planteado en la introducción, en nuestra nómina de gramáticas nos interesa analizar de manera especial tres aspectos: (i) si se incluye información sobre formación de palabras, (ii) el lugar en el que se describen tales procedimientos dentro de cada uno de los tratados y (iii) qué mecanismos de formación se reconocen o se intuyen en los textos.

Primeramente, es fundamental comprobar si existen datos sobre formación de palabras en los tratados gramaticales de nuestro corpus a fin de analizar y valorar la teoría propuesta en cada caso.

En segundo lugar, nos parece igualmente relevante observar en qué parte del texto gramatical se ubica la información sobre formación de palabras, pues, como indicamos en Torres (2009), de una parte, en la tradición académica no se localiza un capítulo dedicado de forma íntegra a tal cuestión hasta la GRAE (1920) y, de otra parte, como hemos señalado más arriba, el primer tratado dedicado a la formación de palabras por un autor ajeno a la Corporación se publica por vez primera en 1917 (Tratado de formación de palabras de Alemany Bolufer).

En cuanto a la tercera cuestión, teniendo en cuenta los procesos de formación de palabras estudiados habitualmente en la tradición gramatical (composición, derivación y parasíntesis), creemos esencial comprobar si todos ellos se reconocen explícitamente o si, al menos, se intuyen en la selección de gramáticas decimonónicas que examinamos.

\subsection{Contexto académico}

En lo que respecta a la obra gramatical académica, hemos seleccionado aquellas ediciones decimonónicas consideradas clave desde el punto de vista de la innovación doctrinal en los trabajos de Gómez Asencio y Garrido (2005: 599) y Gómez Asencio (2008: 38), esto es, las GRAEs (1854, 1858, 1870, 1874 y 1880).

A continuación, en la tabla de (1) presentamos la información sobre formación de palabras documentada en las gramáticas académicas analizadas.

A la vista de (1), comprobamos a priori que en los textos académicos analizados hallamos datos sobre formación de palabras a la hora de describir algunas partes de la oración.

En concreto, la información que nos interesa queda ubicada en los capítulos relativos al nombre, adjetivo ${ }^{7}$ y verbo, todos ellos incluidos en la prime-

7 A partir de 1870 la Academia considera el sustantivo y el adjetivo como clases autónomas y, por consiguiente, les dedica sendos capítulos. En ediciones anteriores las GRAEs agrupan sustantivo y adjetivo bajo la denominación nombre: "Tan inseparables suelen andar el 


\begin{tabular}{|c|c|c|}
\hline & \multicolumn{2}{|c|}{$\begin{array}{l}\text { INFORMACIÓN SOBRE FORMACIÓN DE PALABRAS } \\
\text { CONTEXTO ACADÉMICO }\end{array}$} \\
\hline \multirow{8}{*}{ GRAEs (1854 y 1858) } & \multirow{7}{*}{$\begin{array}{l}\text { De las varias especies y } \\
\text { diferencias de nombres } \\
\text { [sustantivo y adjetivo] } \\
\text { (cap. V de la Analogia) }\end{array}$} & $\begin{array}{c}\text { Primitivos } \\
(\text { cielo })\end{array}$ \\
\hline & & $\begin{array}{c}\text { Derivados (celestial): } \\
\text { gentilicios o nacionales (leonés), } \\
\text { patronímicos (Álvarez), aumentativos } \\
\text { (cucharón), diminutivos (chiquillo) }\end{array}$ \\
\hline & & $\begin{array}{c}\text { Colectivos } \\
\text { (ejército) }\end{array}$ \\
\hline & & $\begin{array}{l}\text { Verbales } \\
\text { (andadura) }\end{array}$ \\
\hline & & $\begin{array}{c}\text { Compuestos } \\
\text { (cuellicorto, sobrepuesto) }\end{array}$ \\
\hline & & $\begin{array}{l}\text { Positivos, comparativos y superlativos } \\
\text { (malo, peor, malísimo) }\end{array}$ \\
\hline & & $\begin{array}{c}\text { Numerales: } \\
\text { absolutos o cardinales (uno), ordinales } \\
\text { (primero), colectivos (docena) y partitivos } \\
(\text { tercio }) \\
\end{array}$ \\
\hline & $\begin{array}{c}\text { De los verbos } \\
\text { impersonales, defectivos, } \\
\text { compuestos y } \\
\text { frecuentativos } \\
\text { (cap. X de la Analogía) }\end{array}$ & $\begin{array}{l}\text { Simples y compuestos } \\
\text { (tener y contener) }\end{array}$ \\
\hline \multirow{11}{*}{ GRAE (1870) } & \multirow{5}{*}{$\begin{array}{c}\text { De las varias especies de } \\
\text { nombres } \\
\text { (sección dentro del cap. II, } \\
\text { Del nombre, de la } \\
\text { Analogía) }\end{array}$} & Primitivos (monte) y derivados (montero) \\
\hline & & $\begin{array}{c}\text { Simples (discipulo) y compuestos } \\
\text { (condiscipulo, quitaipón })\end{array}$ \\
\hline & & $\begin{array}{c}\text { Aumentativos (zagalón) y diminutivos } \\
\text { (frailecito) }\end{array}$ \\
\hline & & Colectivos (enjambre) \\
\hline & & Verbales (hacedor) \\
\hline & \multirow{5}{*}{$\begin{array}{c}\text { De las varias especies de } \\
\text { adjetivos } \\
\text { (sección dentro del cap. } \\
\text { III, Del adjetivo, de la } \\
\text { Analogía) }\end{array}$} & $\begin{array}{c}\text { Primitivos (azul) y derivados (azulado, } \\
\text { azulino) }\end{array}$ \\
\hline & & Simples (útil) y compuestos (inútil) \\
\hline & & $\begin{array}{c}\text { Positivos (bueno), comparativos (mejor) y } \\
\text { superlativos (blandisimo) }\end{array}$ \\
\hline & & Verbales (amable) \\
\hline & & Numerales (uno, postrer) \\
\hline & $\begin{array}{c}\text { De los verbos } \\
\text { impersonales, defectivos y } \\
\text { compuestos } \\
\text { (cap. VII de la Analogía) }\end{array}$ & Simples (meter) y compuestos (entremeter) \\
\hline \multirow{5}{*}{ GRAEs (1874 y 1880$)$} & \multirow{5}{*}{$\begin{array}{c}\text { De las varias especies de } \\
\text { nombres } \\
\text { (sección dentro del cap. II, } \\
\text { Del nombre, de la } \\
\text { Analogía) } \\
{[G R A E, 1874]} \\
--- \\
\text { De las varias especies de } \\
\text { nombres } \\
\text { (sección dentro del cap. }\end{array}$} & Primitivos (pan) y derivados (panadero) \\
\hline & & Simples (fino) y compuestos (entrefino) \\
\hline & & Colectivos (veintena) \\
\hline & & Partitivos (mitad) \\
\hline & & Proporcionales (duplo) \\
\hline
\end{tabular}




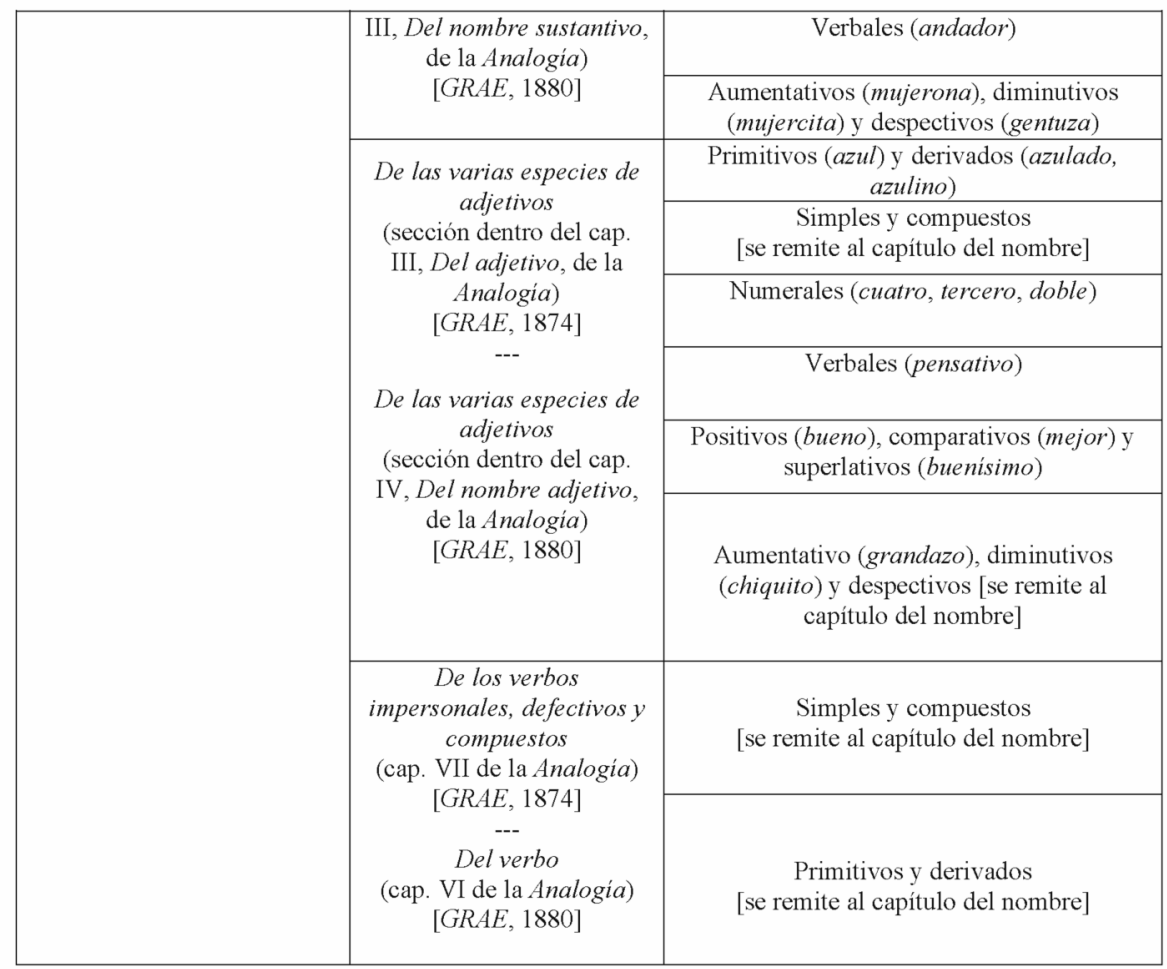

Tabla 1. Información sobre formación de palabras en gramáticas académicas

ra parte del texto, la Analogía ("enseña el valor gramatical de las palabras con todos sus accidentes y propiedades", GRAE, 1858: 1) ${ }^{8}$. Como hemos comentado más arriba, habrá que esperar hasta la GRAE (1920) para que la Academia incluya como novedad en su texto gramatical un capítulo íntegramente dedicado a la formación de palabras (De la formación de palabras. La derivación, la composición y la parasíntesis).

Aunque en la tabla presentamos las subclases de nombre, adjetivo y verbo distinguidas por las GRAEs, es necesario señalar que en nuestro estudio no pretendemos ofrecer un análisis pormenorizado desde el punto de vista for-

Adjetivo y el Substantivo [...] que no anduvieron del todo desacertados los primeros gramáticos al incluirlos en un solo grupo" (GRAE, 1870: XIV). En este sentido, Gómez Asencio (1981: 126) indica que "fueron los gramáticos escolásticos medievales del siglo XII (Abelardo, Pedro Helías) los primeros que llevaron a cabo una división del nombre en esas dos subclases".

8 Ya Suárez (1999: 628), al analizar los aspectos relacionados con la formación de palabras en la gramática de Nebrija, apuntaba que "este tema no aparece tratado autónomamente sino vinculado a otros aspectos, concretamente a los accidentes del nombre y del verbo". 
mal y semántico (cfr. Gómez Asencio, 1985; Calero, 1986; Ramajo, 1987; Lliteras, 1992; Hernando, 2006), sino que nos interesa comprobar la presencia de la teoría sobre formación de palabras, a saber, si esta cuestión alcanza suficiente entidad como para codificarse a modo de sección o capítulo, circunstancia que, según comprobamos en la tabla (1), no se da en las gramáticas académicas consultadas. No obstante, presentamos la clasificación de las correspondientes subclases a fin de comprobar y valorar qué proceso alcanza un mayor protagonismo (derivación, composición o parasíntesis).

En lo que respecta a la información sobre formación de palabras documentada a propósito del nombre y del adjetivo, observamos que esta se ubica al hablar de las especies de sendas clases de palabras (capítulos De las varias especies y diferencias de nombres en GRAEs, 1854 y 1858; De las varias especies de nombres y De las varias especies de adjetivos en GRAEs, 1870, 1874 y 1880). En cambio, en el caso del verbo, tal cuestión se localiza en el capítulo dedicado a los Verbos impersonales, defectivos y compuestos (GRAEs, 1858-1874) y en el relativo al Verbo (GRAE, 1880).

En concreto, en las GRAEs (1854 y 1858) las subclases de nombre distinguidas por la Corporación son las siguientes: primitivos, derivados (gentilicios o nacionales; patronímicos; aumentativos; diminutivos), colectivos; verbales; compuestos; positivos, comparativos y superlativos; numerales (cardinales; ordinales; colectivos; partitivos).

En cambio, a partir de la GRAE (1870), cuando sustantivo y adjetivo se separan en partes de la oración independientes, se produce un reajuste y las subclases reconocidas hasta el momento se distribuyen en sendos capítulos de tal modo que, por un lado, entre las subclases de nombres documentamos los primitivos y derivados, simples y compuestos, aumentativos y diminutivos ${ }^{9}$, colectivos y verbales, a los que hay que añadir los partitivos y los proporcionales a partir de la GRAE (1874). Por otro lado, las subclases reconocidas para el adjetivo en la GRAE (1870) son los primitivos y derivados; simples y compuestos; positivos, comparativos y superlativos; verbales y numerales, a las que se unen los aumentativos, diminutivos y despectivos a partir de la GRAE (1874).

En lo que respecta a los tipos de verbos, hemos de destacar que en las GRAEs (1854, 1858, 1870 y 1874) únicamente se atiende a la clasificación en simples y compuestos, si bien, a partir de la GRAE (1880) la Academia reconoce igualmente la distinción entre primitivos y derivados.

9 Debemos señalar que, aunque no son reconocidos oficialmente como subclase hasta 1874 , los llamados despectivos se integran al final de la explicación de los aumentativos y diminutivos a partir de la GRAE (1854). Además, en lo que respecta a las etiquetas documentadas para aludir a los despectivos, en 1874 se emplea novedosamente el sinónimo menospreciativo, frente a la edición de 1870, en la que se documenta como alternativa la denominación despreciativo. 
A propósito de la clasificación del nombre en la GRAE (1771), que no varía en 1854 y 1858, Gómez Asencio (1985: 13-14) señala la mezcla de criterios (formal y semántico) al caracterizar las distintas subclases, lo que "provoca que los conceptos incluidos dentro de esta clasificación no sean mutuamente excluyentes y que, por ejemplo, desmontable sea a la vez derivado, verbal y positivo". Parece que este fenómeno es habitual en la época, pues, a juicio de Gómez Asencio (1985: 14):

Estos autores no piensan propiamente en clasificaciones, al menos en el sentido en que entendemos hoy este término ${ }^{10}$, sino más bien en enumeraciones de las subclases, listas no sistematizadas en las cuales cada subclase no tiene ninguna relación con las demás subclases y sí solo con la clase en la que está integrada.

No obstante, más adelante, a favor de que los gramáticos pensaban realmente en clasificaciones, Gómez Asencio (1985: 14) apunta lo siguiente:

Cada uno de ellos esboza un nuevo modelo de clasificación con la particularidad de que en él pueden aparecer o no nuevos elementos que añadir a la lista de las subclases, pero siempre sucede que algunos elementos que ya aparecían en listas anteriores son colocados en lugares distintos. Esto podría ser síntoma de la preocupación por corregir las insatisfactorias clasificaciones anteriores y por elaborar una clasificación (en sentido actual) nueva, más adecuada, no una enumeración en la que el lugar ocupado por los elementos no es pertinente.

Tras comentar las subclases de nombres, adjetivos y verbos reconocidas en las GRAEs analizadas, queda patente que la derivación ocupa más páginas que la composición, entendida esta hasta bien entrado el siglo XX como el procedimiento bajo el que se engloba, además de las formaciones creadas mediante la unión de dos palabras independientes (perdonavidas, hazmerreír, ganapierde, etc.), aquellas en las que se anteponen prefijos (anteiglesia, citramontano, infraescrito, etc. $)^{11}$. En cuanto a la parasíntesis, al explicar los nombres compuestos en la GRAE (1880), destacamos un párrafo en el que se intuye la especial estructura de palabras creadas a partir de este procedimiento: "desgarbado y congeniar han de estimarse juntamente derivadas y compuestas, porque se derivan de otras, y porque se componen de elementos distin-

10 Para Gómez Asencio (1985: 14, nota 2) una clasificación propiamente dicha ha de cumplir tres características: "(i) debe usarse un solo criterio clasificatorio; (ii) los términos incluidos en ella, definidos por un mismo criterio, deben ser mutuamente excluyentes; (iii) dichos términos deben entrar en algún sentido, en oposición entre sí".

11 De este modo, en palabras de Montero (1998: 295, nota 5), "la tendencia general en los primeros estudios era la de incluir los prefijos dentro de la categoría preposicional y analizarlos como auténticas preposiciones, frente a la postura actual que acepta que los prefijos no son preposiciones, sino elementos diferentes". 
tos y separables" (GRAE, 1880: 33). No obstante, el término parasíntesis no se documentará en la tradición académica hasta la GRAE (1920)12.

En definitiva, podemos afirmar que las GRAEs atienden preferentemente a explicar el empleo de los sufijos, en especial, el uso de los apreciativos (aumentativos, diminutivos y despectivos) ${ }^{13}$.

\subsection{Contexto no académico}

En cuanto a los textos gramaticales concebidos por autores ajenos a la Academia, analizamos las siguientes obras: Gramática de la lengua castellana según ahora se habla (1830) de Vicente Salvá, Gramática de la lengua castellana destinada al uso de los americanos (1847) de Andrés Bello, Tratado de gramática razonada con aplicación decidida y constante al estudio del idioma español (1885) de Gregorio Herrainz, Gramática razonada de la lengua española (1876) de Matías Salleras y Tratado elemental de la lengua castellana o española (1896) de Rufino Blanco y Sánchez.

Huelga justificar el motivo por el que, al seleccionar el siglo XIX como etapa cronológica objeto de estudio, analizamos los tratados de Salvá y Bello, pues de todos es sabido que suponen hitos en la historia de las ideas gramaticales $^{14}$. Los demás textos no académicos incluidos en nuestra nómina responden a una tipología heterogénea: los de Herrainz y Blanco se destinan a alumnos de primera enseñanza ${ }^{15}$, mientras que la gramática de Salleras, de orienta-

12 Además de incluir como novedad el capítulo sobre formación de palabras (cap. IX) -en el que se explican los procedimientos de composición, derivación y parasíntesis-, la GRAE (1920) ofrece información sobre esta materia en el capítulo del nombre sustantivo, en el que clasifica esta clase de palabra, según su especie, en simple, compuesto y parasintético: "Se llama simple el vocablo a cuya formación no contribuye ninguna otra voz agregada; v. gr. coro, fino, tener, donde; compuesto, el que consta de una palabra simple y de otra u otras voces o partículas, como ante-coro, entre-fino, con-tener, a-donde; y parasintético, el que a la vez es derivado y compuesto, como misacantano, endulzar, etc." (GRAE, 1920: 21).

13 Según indica González Ruiz (1994: 274), "tanto la gradación [superlativos y comparativos] como la sufijación apreciativa, ya desde Dionisio de Tracia, quedaron incluidas en el accidente de la especie junto a los demás sufijos derivativos".

14 El tratado de Salvá es considerado una "gramática española-desde la perspectiva de la época- moderna, renovadora, que -en perspectiva de la época- supera y mejora desde los puntos de vista descriptivo de una norma y teórico lo que la Academia del siglo XVIII había legado" (Gómez Asencio, 2009: 2). Por su parte, la gramática de Bello representa "los textos gramaticales concebidos desde el principio por y para los recién independizados americanos, gramáticas desvinculadas en la medida de lo posible de las tradiciones peninsulares y con los menores anclajes que se pudieran en la oficialidad académica" (Gómez Asencio, 2009: 2).

15 Cabe destacar que Herrainz (2001[1885]: XV) se opone al privilegio que posee el texto gramatical académico de erigirse como única gramática oficial. En cambio, debido probablemente a motivos comerciales, Blanco (1926[1896]: VI) señala en la Advertencia a su tratado la consonancia existente entre la teoría gramatical plasmada en su obra y la de la gramática de la RAE. 
ción logicista, asimila fundamentos racionalistas tales como la defensa de la íntima correspondencia entre lenguaje y pensamiento (cfr. Calero, 1985: 9).

Seguidamente, como en el caso de las GRAEs, en la tabla de (2) presentamos la información concerniente al tratamiento de la formación de palabras en los textos gramaticales no académicos:

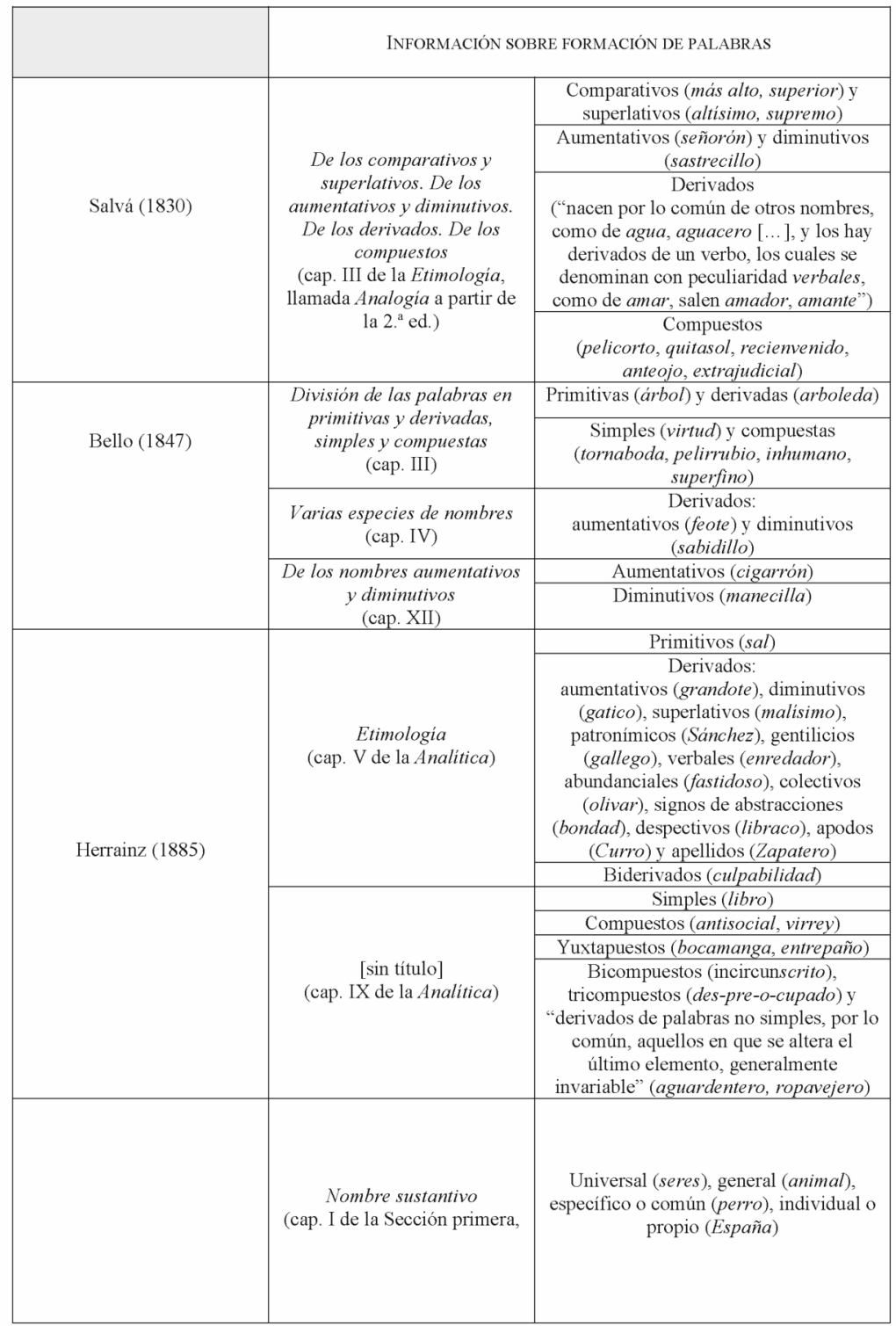




\begin{tabular}{|c|c|c|}
\hline \multirow{9}{*}{ Salleras (1876) } & \multirow{3}{*}{$\begin{array}{c}\text { Palabras sustantivas } \\
\text { [nombre y pronombres], de la } \\
\text { Analogia })\end{array}$} & \multirow{3}{*}{$\begin{array}{l}\text { Universal (seres), general (animal), } \\
\text { especifico o común (perro), individual o } \\
\text { propio (España) } \\
\\
\text { Primitivos (casa) y } \\
\text { derivados: } \\
\text { colectivos (muchedumbre), compuestos } \\
\text { (carricoche, parabien), nacionales o } \\
\text { gentilicios (andaluz), aumentativos } \\
\text { (papelote), diminutivos (hijito) y } \\
\text { despreciativos (santurrón) }\end{array}$} \\
\hline & & \\
\hline & & \\
\hline & & $\begin{array}{l}\text { Primitivos (bueno, grande) y derivados } \\
\text { (bonisimo, grandecito) }\end{array}$ \\
\hline & & $\begin{array}{l}\text { Simples (bueno) y compuestos } \\
\text { (verdinegro, encubierto) }\end{array}$ \\
\hline & $\begin{array}{c}\text { Del adjetivo } \\
\text { (cap. II de la Sección }\end{array}$ & $\begin{array}{c}\text { Aumentativos (blancote) y diminutivos } \\
\text { (blanquecito) }\end{array}$ \\
\hline & $\begin{array}{l}\text { segunda, Palabras } \\
\text { modificativas } o\end{array}$ & $\begin{array}{l}\text { Positivos, superlativos (blanquisimo) } \\
\text { comparativos (superior) }\end{array}$ \\
\hline & $\begin{array}{c}\text { [adjetivo, artículo y } \\
\text { adverbio], de la Analogía) }\end{array}$ & $\begin{array}{c}\text { Verbales (procedente) ["muchos deben } \\
\text { llamarse nominales por derivarse de } \\
\text { nombre"], gentilicios (franceses) y } \\
\text { abundanciales (enfermizo) }\end{array}$ \\
\hline & & $\begin{array}{c}\text { Cualitativos (verde), cuantativos (dos, } \\
\text { pocos), relativos (cuyo, mio) y } \\
\text { circunstanciales (temprano) }\end{array}$ \\
\hline \multirow{7}{*}{ Blanco (1896) } & \multirow[b]{2}{*}{$\begin{array}{l}\text { Nombre sustantivo } \\
\text { (cap. II de la Analogia) }\end{array}$} & $\begin{array}{c}\text { Simples (campo) y compuestos } \\
\text { (camposanto) }\end{array}$ \\
\hline & & $\begin{array}{l}\text { Primitivos (casa) y derivados (casero) } \\
\text { [tipos de derivados: aumentativos, } \\
\text { diminutivos, despectivos, patronímicos y } \\
\text { verbales] }\end{array}$ \\
\hline & \multirow{2}{*}{$\begin{array}{l}\text { Nombre adjetivo } \\
\text { (cap. II de la Analogia) }\end{array}$} & $\begin{array}{l}\text { Simples y compuestos } \\
\text { [se remite al capitulo del nombre] }\end{array}$ \\
\hline & & $\begin{array}{c}\text { Primitivos y derivados } \\
\text { [tipos de derivados: aumentativos, } \\
\text { diminutivos, despectivos y verbales] }\end{array}$ \\
\hline & $\begin{array}{l}\text { Composición de las palabras } \\
\text { (cap. III de la Lexigrafia) }\end{array}$ & $\begin{array}{c}\text { Puntapié, metesillas, representación, } \\
\text { independiente }\end{array}$ \\
\hline & $\begin{array}{c}\text { De la derivación de las } \\
\text { palabras } \\
\text { (cap. IV de la Lexigrafia) }\end{array}$ & $\begin{array}{c}\text { "sufijos aumentativos" (-acho, -ote) } \\
\text { "sufijos diminutivos" (-iño, -uelo }) \\
\text { "los que se unen a las raíces de verbos } \\
\text { para formar sustantivos y adjetivos" } \\
\text { (-ación, -ble, -dor) }\end{array}$ \\
\hline & $\begin{array}{l}\text { De la parasintesis } \\
\text { (cap. V de la Lexigrafia) }\end{array}$ & Desalmado, aprisionar \\
\hline
\end{tabular}

Tabla 2. Información sobre formación de palabras en gramáticas no académicas

En la tabla de (2) comprobamos que en las gramáticas ajenas a la Corporación también se incluyen cuestiones sobre formación de palabras. En cuanto al lugar en el que se localizan tales datos, en relación con las GRAEs, comprobamos que en la tradición no académica los aspectos sobre 
formación de palabras quedan contenidos en diversos capítulos y no solo al tratar el nombre, el adjetivo y el verbo.

De este modo, atendiendo a los datos presentados en la tabla de (2), podemos secuenciar los textos en tres grupos:

i. Gramáticas que, como en el caso de las GRAEs, únicamente ubican los datos sobre formación de palabras en capítulos referidos a las distintas partes de la oración (en concreto, el sustantivo y el adjetivo): Salleras (1876).

ii. Gramáticas que, además de documentar aspectos sobre formación de palabras en los capítulos concernientes al sustantivo y al adjetivo, incluyen algunas cuestiones en capítulos dedicados exclusivamente a los distintos mecanismos de formación de palabras: Bello (1847) y Blanco (1896).

iii. Gramáticas en las que solo se localizan datos sobre formación de palabras en capítulos destinados íntegramente a tal cuestión: Salvá (1830) y Herrainz (1885).

Seguidamente, atendiendo a los tres grupos creados más arriba, presentamos los datos sobre formación de palabras incluidos en los tratados examinados. Como en el caso del contexto académico, aunque ofrecemos las distintas subclases reconocidas por los gramáticos, no aspiramos a comentar tal clasificación de manera detallada, pues únicamente pretendemos comprobar qué procedimiento de formación de palabras adquiere más peso en la teoría gramatical decimonónica.

En cuanto al texto gramatical ubicado en el primer grupo, esto es, la Gramática razonada (1876) de Salleras, en ella la información relativa a la formación de palabras queda ubicada en la Analogía ("se ocupa de las palabras o partes de la oración", Salleras, 2001[1876]: 33), como en el caso de las GRAEs. En concreto, hallamos tales datos en dos de las cinco secciones que componen esta parte de la Gramática: Palabras sustantivas (capítulo del Nombre sustantivo) y Palabras modificativas (capítulo Del adjetivo). Tanto en la clasificación del sustantivo como en la del adjetivo observamos que se produce una mezcla de los criterios semántico y formal al distinguir las diferentes subclases ${ }^{16}$, tal como anunciaba Gómez Asencio (1985: 13-14) en el caso de las GRAEs y como veremos en los demás tratados que analizaremos a continuación. A la vista de la tipología establecida por Salleras, queremos destacar el hecho de que, en el caso de los sustantivos -debido probablemente a un error-se incluyen los compuestos ${ }^{17}$

16 Por un lado, atendiendo al criterio semántico, se habla de sustantivos universales, generales, comunes, propios y de adjetivos cualitativos, cuantativos, relativos, circunstanciales. Por otro lado, desde una perspectiva formal, se distinguen, por ejemplo, sustantivos y adjetivos primitivos y derivados.

17 Los nombres compuestos, frente a los simples ("los que solo constan de una palabra", hombre, rayo, coche), son "todos los que están formados de dos o más palabras del idioma 
como subclase de derivados ("los nombres colectivos, los compuestos, los gentilicios y muchos aumentativos y diminutivos deben considerarse como nombres derivados", Salleras, 2001[1876]: 49). No obstante, si nos centramos en las subclases de adjetivos, comprobamos que el autor no agrupa a los derivados como lo hiciera a propósito del sustantivo, sino que va presentando los distintos tipos y, tras la definición de derivado, comenta que "muchas de las variedades que a continuación exponemos pertenecen al grupo de los derivados" (Salleras, 2001[1876]: 78) ${ }^{18}$.

En segundo lugar, en lo que respecta a los tratados agrupados en (ii), esto es, los publicados por Bello y Blanco, en ellos localizamos aspectos sobre formación de palabras tanto en capítulos dedicados a explicar las clases de palabras como en capítulos ya destinados a presentar una tipología de compuestos y derivados (en Bello y en Blanco) y parasintéticos (solo en el caso de Blanco).

Por su parte, Bello incluye en su gramática un capítulo titulado División de las palabras en primitivas y derivadas, simples y compuestas (cap. III) ${ }^{19}$, en el que el venezolano expone su teoría sobre formación de palabras, centrándose sobre todo en los compuestos y, de manera especial, en las partículas compositivas que entran a formar parte de este tipo de voces ${ }^{20}$. Además, Bello alude a otras cuestiones sobre formación de palabras. En concreto, se ocupa de los aumentativos y los diminutivos, tanto en el capítulo IV (Varias especies de nombres ${ }^{21}$ ), en el que avanza las definiciones de los derivados aumentati-

a que pertenecen o de una del idioma y otra extranjera" (carri-coche, para-rayos, prohombre [sic]), tal como explica Salleras (2001[1876]: 50). Al observar las partes de la oración que forman los compuestos, este gramático reconoce que en la mayoría de los casos una preposición — castellana, latina o griega - se antepone al nombre (condiscípulo, contrapunto, indolencia, parabién, preposición, subterfugio), si bien también documenta compuestos creados a partir de "dos nombres" (puntapie, varapalo), "verbo y nombre" (paraguas, quitasol), "adverbio y nombre" (bienandanza, malquerencia), e incluso, de "dos o tres verbos y otra palabra más" (corre-ve-di-le, gana-pierde, va-i-ven).

18 Aunque no se alude a los tipos de derivados de manera explícita, gracias a los ejemplos que presenta al definir los derivados (grandecito, bonísimo), entendemos que los diminutivos y los superlativos se incluyen bajo este grupo. Además, tanto al explicar los aumentativos y diminutivos como los verbales y abundanciales, define estos tipos como derivados.

19 A diferencia de los demás gramáticos, Bello no concibe divisiones en la Gramática (cfr. Gómez Asencio, 1981: 33), por lo que sus capítulos no se insertan en partes concretas.

20 Bello (1988[1847]: 197-198) diferencia dos tipos de partículas compositivas: las separables - "se usan también como palabras independientes", a-, ante-, con-, en-y las inseparables - que "eran en aquella lengua [latina] dicciones independientes", abs-, anti-, extra, retro-- Esta distinción muestra que Bello, como la mayoría de los gramáticos coetáneos, no identifica las preposiciones que empleamos en español con aquellas unidades que, si bien son formalmente idénticas, forman palabras complejas, a pesar de que incluye la prefijación dentro de la composición.

21 Bello engloba al sustantivo y al adjetivo bajo el nombre ("los nombres son sustantivos o adjetivos", Bello, 1988[1847]: 205). 
vos y diminutivos, como en el XII (De los nombres aumentativos y diminutivos), en el que se ocupa de estas subclases de manera extensa.

Asimismo, en el Tratado de Blanco hemos localizado cuestiones sobre formación de palabras en cinco capítulos, dos incluidos en la Analogía (los relativos al nombre sustantivo y al nombre adjetivo, a los que se le reconocen las subclases primitivo, derivado, simple y compuesto) y tres en la denominada Lexigrafía ${ }^{22}$ (los concernientes a los mecanismos de composición, derivación y parasíntesis, capítulos destinados íntegramente a la formación de palabras).

Además de indicar que Blanco también considera compuestos aquellos vocablos creados a partir de prefijos (por ejemplo, independiente), es interesante, y a la vez novedoso, destacar su capítulo sobre parasíntesis, al ser el único autor de nuestro corpus que alude explícitamente a este procedimiento de formación de palabras ${ }^{23}$. Para Blanco (1926[1896]: 329) la parasíntesis es "la manera de formar las palabras por composición y derivación a la vez" -picapedrero (pica-pe-dr-ero) y endulzar (en-dulz-ar)_- ${ }^{24}$.

Finalmente, nos ocupamos del tercer grupo de gramáticas, a saber, las publicadas por Salvá (1830) y Herrainz (1885), en las que se localizan capítulos dedicados exclusivamente a la formación de palabras.

22 Blanco (1926[1896]: 294) explica que el contenido de la llamada Lexigrafía, parte de la Gramática dedicada al estudio de la significación de los vocablos, se puede dividir en cuatro partes: (i) el estudio de la significación de las palabras por su origen (Etimología), atendiendo principalmente a los sonidos (Fonología) y a la forma (Morfología); (ii) el estudio de la significación de las palabras por el uso autorizado en el Diccionario; (iii) el estudio de los modismos e idiotismos y, finalmente, (iv) el estudio de los dichos populares (Folklore) y de los refranes y proverbios (Paremiología). Tal como señala Calero (1986: 273), para otros gramáticos del siglo XIX tales como Fernández Monje, Ovalle y Flórez la Lexigrafía se puede definir como la parte de la Gramática que estudia "la significación de las palabras homónimas y sinónimas; arcaísmos y neologismos"; "las palabras, sus accidentes y propiedades, las frases y partículas" y "las acepciones individuales de los vocablos, según el valor recto, extensivo y translaticio", respectivamente.

23 Según señala Serrano-Dolader (1999: 4701), Darmesteter (1967[1875]: 96-97) es el primer autor que desarrolla el concepto de parasíntesis - "le résultat d'une composition et d'une dérivation agissant ensemble sur un même radical, de telle sorte que l'une ou l'autre ne peut pas être supprimée sans amener la perte du mot"-, a propósito de los compuestos creados a partir de una partícula y de un nombre o adjetivo que dan lugar a verbos (barque > em-barqu-er, dé-barqu-er).

$24 \mathrm{Al}$ considerar la prefijación dentro de la composición, el autor presenta como ejemplo de parasintéticos tanto picapedrero como endulzar. Actualmente, en teoría morfológica se contemplan dos tendencias a la hora de considerar palabras como picapedrero o ropavejero: (i) catalogarlas como compuestos con derivación externa (cfr. Varela, 2005: 34-35, nota 3) o (ii) como parasintéticos. A su vez, debido a que el término parasíntesis se refiere a un proceso morfológico en el que intervienen dos procedimientos de formación de palabras simultáneamente, en la actualidad se documenta una doble concepción de parasíntesis: (i) cuando prefijo y sufijo se aplican a la vez sobre la base de derivación (aterrizar, encarecer) y (ii) cuando hay síntesis de derivación y composición (picapedrero, ropavejero) (cfr. Serrano-Dolader, 1999: 4701). 
Salvá presenta en la primera parte de su obra -llamada Etimología en la edición princeps y Analogía a partir de la segunda (cfr. Lliteras, 1988: 37)- un capítulo denominado De los comparativos y superlativos. De los aumentativos $y$ diminutivos. De los derivados. De los compuestos ${ }^{25}$.

En lo que respecta a los aumentativos y diminutivos, el gramático valenciano se centra en listar las "terminaciones" que los forman y sus significados. En cuanto a los derivados, Salvá señala especialmente los que proceden de nombres (aguacero y aguada, de agua) y aquellos que nacen de verbos, a los que denomina verbales (amador, amante, amado, de amar). En el caso de los compuestos, como lo hiciera Bello, Salvá se ocupa de explicar la naturaleza de estas formaciones (dos nombres, aguachirle; nombre y verbo, perdonavidas; nombre y preposición, concuñado; etc.), así como de especificar las partículas componentes que pueden emplearse a la hora de crear compuestos (ab-, anti-, circum-, entre-, pro-, re-, sobre-, etc.).

Al estudiar el tratamiento de la formación de palabras en la obra de Salvá, Henríquez (2002: 223-227) destaca la modernidad de este gramático en lo que respecta a la teoría sobre los diminutivos y aumentativos, a los que dedica un extenso apartado en el que, por ejemplo, se señala la lexicalización de formaciones como azucarillo, gusanillo, pastilla, peluquín, etc.

En cuanto al Tratado de Herrainz, las cuestiones sobre formación de palabras quedan ubicadas en dos capítulos integrados en la primera parte de la obra, la Analítica ${ }^{26}$. De una parte, en el capítulo denominado Etimología, que se ocupa "del origen y forma de los vocablos de un idioma" (Herrainz, 2001 [1885]: 33) y donde se distingue entre primitivos, derivados y biderivados ("palabra que resulta de otra ya derivada: culpabilidad, de culpable", Herrainz, 2001[1885]: 36). De otra parte, en el capítulo IX, sin título, en el que se caracterizan los simples, compuestos, yuxtapuestos -procedimiento en el que los vocablos conservan su acento, aunque se les una en la escritura, boca-manga, entre-paño- y bicompuestos (incircunscrito), tricompuestos (despre-o-cupado) y derivados de palabras no simples -"por lo común, aquellos en que se altera el último elemento, generalmente invariable" (aguardentero, ropavejero)-. En estas últimas formaciones reconocemos la estructura del procedimiento llamado por Blanco parasintesis, denominado por Herrainz derivación de palabras no simples.

25 No obstante, debemos destacar que, a partir de la segunda edición de la obra, en el capítulo II (De las partes de la oración en general y del nombre en particular. De sus números y géneros. De los adjetivos), tras la definiciones del nombre sustantivo y del nombre adjetivo, se incluye un párrafo extenso en el que se presentan las subclases de nombres (propio, apelativo, colectivo, verbal, simple, compuesto, aumentativo y diminutivo).

26 A su vez, dividida en tres partes: Analogía, Etimología y Lexicología. 


\subsection{Tradición académica vs. tradición no académica}

En lo que respecta a la formación de palabras, los textos de nuestro corpus presentan un tratamiento dispar, según hemos comprobado en los apartados anteriores.

Seguidamente, disponemos la información siguiendo una ordenación epistémica, esto es, agrupamos los tratados analizados atendiendo a los posibles saltos doctrinales localizados en la teoría sobre formación de palabras que presenta cada gramático.

Como ya procedimos en el contexto no académico, el criterio en el que nos basamos para organizar epistémicamente los tratados es si existe un apartado o capítulo dedicado íntegramente a la formación de palabras.

En virtud de este parámetro, podemos clasificar los textos en tres grupos:

i. Presentación dispersa: comentarios sobre formación de palabras dentro de los capítulos dedicados a cada clase de palabras: GRAEs (1854, $1858,1870,1874$ y 1880) y Salleras (1876).

ii. Presentación mixta: mezcla de comentarios y secciones sobre la formación de cada clase de palabras con un capítulo o apartado dedicado exclusivamente a la formación de palabras: Bello (1847) y Blanco (1896).

iii. Presentación unificada: se documenta exclusivamente un capítulo o apartado que integra todos los aspectos sobre los procedimientos de formación de palabras: Salvá (1830) y Herrainz (1885).

En lo que respecta a la ubicación de la información sobre formación de palabras, en la mayoría de los casos queda recogida bajo la primera parte de las gramáticas, a saber, la Analogía (GRAEs, Salvá, Salleras, Blanco). No en vano, según señala Gómez Asencio (1981: 36):

La Analogía es entendida por los gramáticos como el estudio total, es decir, en todos sus aspectos, de las palabras o partes de la oración tomadas separadamente; o, mejor dicho, como el estudio sincrónico de las palabras: definición, clasificación y características de cada una de las clases de palabras; flexión y formación (composición y derivación) de las mismas, prestando más atención a las propiedades formales de las palabras que a las funcionales o morfofuncionales.

En cuanto a los mecanismos reconocidos, tras analizar los distintos tratados gramaticales, sintetizamos las cuestiones clave:

i. A la vista de las distintas subclases de nombres, adjetivos y verbos y de los capítulos ya dedicados exclusivamente a la formación de palabras, en todos los textos se distinguen explícitamente los procedimientos de composición y derivación. Blanco es el único gramático del corpus que habla de parasíntesis, si bien 
los demás autores incluyen ejemplos de palabras creadas a partir de este procedimiento, aunque aún no las etiquetan como parasintéticas, sino que las incluyen entre los derivados o entre los compuestos ${ }^{27}$. Recordamos que la Academia intuye la especial estructura de palabras como desgarbado o congeniar.

ii. Todos los gramáticos incluyen la prefijación dentro de la composición y no de la derivación ${ }^{28}$, debido básicamente a que algunos de los prefijos empleados para crear nuevas palabras coinciden formalmente con preposiciones del español (ante-, con-, contra-, sobre-, etc.) y otros, aunque carecen de esta similitud con preposiciones de nuestra lengua, proceden etimológicamente de preposiciones grecolatinas (ambi-, epi-, extra-, post-, etc. $)^{29}$.

iii. Al tratar la derivación, la mayoría de los tratados, especialmente los ajenos a la Corporación, se centran fundamentalmente en listar y caracterizar los distintos sufijos diminutivos y aumentativos (en especial, Salvá y Bello). Además, también se suelen ocupar de manera más extensa de los derivados deverbales (andador, pensativo).

\section{Valoraciones finales}

Al analizar el tratamiento de la formación de palabras en una selección de textos gramaticales publicados a lo largo del siglo XIX y adscritos tanto a la tradición académica como a la no académica, hemos arrojado luz acerca de algunas cuestiones fundamentales:

i. Si bien hallamos capítulos dedicados íntegramente a la formación de palabras a partir del segundo cuarto del siglo XIX (Salvá, Bello, Herrainz y Blanco), es necesario insistir en que los gramáticos, siguiendo la tradición grecolatina, incluyen datos al ocuparse de las distintas clases de palabras (en concreto, sustantivos, adjetivos y verbos). En este sentido, a pesar de la mez-

27 A modo de ejemplo, Bello (1988[1847]: 195 y 196) considera al verbo acercar entre los ejemplos de derivados ("el verbo acerco [se deriva] del adverbio cerca"), y al verbo amontonar, entre los compuestos ("amontono, verbo compuesto de la preposición a y el sustantivo montón"). 28 En la tradición gramatical española se ha considerado que las formaciones creadas a partir de prefijos se adscriben al mecanismo de la composición debido fundamentalmente tanto a la coincidencia formal de estos morfemas con preposiciones españolas (ante-, sobre-) o de origen grecolatino (hiper-, super-) como al hecho de que el prefijo, frente al sufijo, no modifica la clase gramatical de la base a la que se adjunta.

29 Felíu (2003: 42-43) constata la indudable relación que existe entre prefijos y preposiciones tanto desde una perspectiva diacrónica como desde un punto de vista sincrónico. Esta autora alude a la identificación de prefijo y preposición por parte de algunos lingüistas actuales y recuerda que habitualmente estos autores distinguen entre preposiciones separables y preposiciones inseparables, etiquetas empleadas por la tradición gramatical española a la hora de caracterizar los prefijos que coinciden formalmente con una preposición del español ( $a-$, en-, entre-, etc.) y los prefijos de origen latino que no poseen correlato preposicional en nuestra lengua (abs-, etc.), respectivamente. 
cla de criterios a la hora de definir las distintas subclases de palabras, resulta significativo el esfuerzo de estos gramáticos por incluir en sus descripciones aspectos que atañen a las variaciones debidas a los procesos de formación de nuevas unidades. Así, tal como apunta Suárez (1999: 642):

Por encima de las incoherencias, destaca la capacidad de los gramáticos para captar rasgos importantes de la lengua que describen, que plasman a veces sólo a través de ejemplos o que, sin lograr discernir bien lo que hay detrás, reflejan en todo caso intuiciones muy certeras.

ii. Atendiendo a la clasificación epistémica presentada más arriba, observamos que todas las gramáticas académicas consultadas se ubican en el primer grupo (presentación dispersa). En cambio, los textos no académicos analizados se distribuyen tanto en la presentación dispersa como en la presentación mixta y en la unificada. En concreto, podemos destacar que la gramática de Salvá (1830), aún siendo la más antigua de nuestra nómina, se alza como la más innovadora en cuanto al tratamiento de la formación de palabras, junto con el tratado de Herrainz (1885). De otro lado, la obra más moderna desde el punto de vista cronológico (Tratado elemental de la lengua castellana o española, 1896, de Blanco) no se localiza entre las gramáticas de presentación unificada, como cabría esperar, sino que se documenta entre los textos de presentación mixta. Por tanto, comprobamos que no existe coincidencia entre la secuencia cronológica y la epistémica.

iii. Aunque en todas las obras quedan reconocidos y caracterizados los procedimientos de composición y derivación, el primer mecanismo se describe generalmente de manera más breve que el segundo, en consonancia con el carácter menos productivo de la composición, bajo la que se engloba la prefijación. En cuanto a la parasintesis, el único gramático que alude explícitamente a este procedimiento de formación de palabras es Blanco, si bien los demás autores incluyen palabras creadas mediante tal mecanismo a la hora de ejemplificar los derivados o los compuestos.

En definitiva, tras examinar y valorar los datos sobre formación de palabras contenidos en una selección de textos gramaticales decimonónicos, queremos destacar la labor de los gramáticos a la hora de describir, sistematizar y elaborar clasificaciones, apegadas a la tradición en algunos casos, dotadas de una incipiente originalidad en otros.

\section{Referencias bibliográficas}

\section{Fuentes primarias}

Bello, A. (1988[1847]): Gramática de la lengua castellana destinada al uso de los americanos. Ed. por Ramón Trujillo. Madrid, Arco Libros. 
Blanco y Sánchez, R. (1926[1896]): Tratado elemental de lengua castellana o española. Madrid, Tipografía de la Revista de Archivos, Bibliotecas y Museos, 8. ${ }^{a}$ ed.

Herráinz y de Heras, G. (1885): Tratado de gramática razonada con aplicación decidida y constante al estudio del idioma español. Segovia, F. Santiuste.

Real Academia Española (1854): Gramática de la lengua castellana. Madrid, Imprenta Nacional.

Real Academia Española (1858): Gramática de la lengua castellana. Madrid, Imprenta Nacional.

Real Academia Española (1870): Gramática de la lengua castellana. Madrid, Imprenta y estereotipia de M. Rivadeneyra.

Real Academia Española (1874): Gramática de la lengua castellana. Madrid, Imprenta y fundación de Manuel Tello.

Real Academia Española (1880): Gramática de la lengua castellana. Madrid, Gregorio Hernando.

Salleras, M. (1876): Gramática razonada de la lengua española. Segovia, Pedro Ondero. Salvá, V. (1988[1830]): Gramática de la lengua castellana según ahora se habla. Ed. por Margarita Lliteras. Madrid, Arco/Libros.

\section{Fuentes secundarias}

Alemany Bolufer, J. (1920): Tratado de la formación de palabras en la lengua castellana. La derivación y la composición. Madrid, Librería General de Victoriano Suárez.

Calero Vaquera, M. ${ }^{a}$ L. (1985): "La Gramática Razonada (1876) de Matías Salleras", Alfinge, 3, págs. 7-20.

Calero Vaquera, M. ${ }^{a}$ L. (1986): Historia de la gramática española (1847-1920). De Andrés Bello a Rodolfo Lenz. Madrid, Gredos.

Darmesteter, A. (1967[1875]): Traité de la formation des mots composés dans la langue française comparée aux autres langues romanes et au latin. París, Librairie Honoré Champion, $10 .^{\mathrm{a}}$ ed.

Felíu Arquiola, E. (2003): Morfología derivativa y semántica léxica: la prefijación de auto-, co- e inter-. Madrid, Universidad Autónoma de Madrid.

García-Medall, J. (1995): Casi un siglo de formación de palabras del español (19001994). Guía bibliográfica. Anejo XIII de la Revista Cuadernos de Filología. Valencia, Universitat de València.

García Platero, J. M. (1998): "Consideraciones sobre la formación de palabras del español”, Estudios de Lingüística. Universidad de Alicante, 12, págs. 69-78.

Gómez Asencio, J. J. (1981): Gramática y categorías verbales en la tradición española (1771-1847). Salamanca, Universidad de Salamanca.

Gómez Asencio, J. J. (1985): Subclases de palabras en la tradición española (17711847). Salamanca, Universidad de Salamanca.

Gómez Asencio, J.. J. (comp.) (2001): Antiguas gramáticas del castellano. Colección Clásicos Tavera, 63, Lingüística y antecedentes literarios de la Península Ibérica, Serie VIII, vol. 1. Madrid, Fundación Histórica Tavera y DIGIBIS [CD-ROM].

Gómez Asencio, J. J. (2008): "El trabajo de la Real Academia Española en el siglo XVIII (y después)", Península. Revista de Estudios Ibéricos, 5, págs. 31-53. 
Gómez Asencio, J. J. (2009): "De gramáticas para americanos a gramática de todos. El caso de Bello (1847)". Revista argentina de historiografía lingüística, I (1), págs. 1-18.

Gómez Asencio, J. J. y G. B. Garrido Vílchez (2005): "Las gramáticas de la RAE en números". En Santos, L. et alii (eds.): Palabras, norma, discurso. En memoria de Fernando Lázaro Carreter. Salamanca, Universidad de Salamanca, págs. 593-604.

González Ruíz, R. (1994): "Apuntes sobre el tratamiento de la formación de palabras en las primeras gramáticas españolas”. En R. Escavy et alii (eds.): Nebrija V centenario (Actas del Congreso Internacional de Historiografía Lingüística), I. Murcia, Universidad de Murcia, págs. 271-288.

Henríquez Salido, M. ${ }^{a}$ C. (2002): "La formación de palabras en la Gramática de la lengua castellana según ahora se habla de Vicente Salvá". En M. Á. Esparza et alii (eds.): SEHL 2001. Estudios de Historiografía Lingüística. Actas del III Congreso Internacional de la Sociedad Española de Historiografía Lingüística (Vigo, 7-10 de febrero de 2001). Hamburgo, Helmut Buske, págs. 213-228.

Hernando García-Cervigón, A. (2006): El grupo del nombre en la Analogía de la GRAE. Madrid, Editorial Complutense.

Lliteras, M. (1988): "Estudio introductorio". Gramática de la lengua castellana según ahora se habla ordenada por Don Vicente Salvá, I. Madrid, Arco Libros, págs. 7-63.

Lliteras, M. (1992): La teoría gramatical de Vicente Salvá. Madrid, Sociedad General Española de Librería.

Montero Curiel, M. ${ }^{a}$ L. (1998): "La formación de palabras en las gramáticas españolas”. En M. Hummel y C. Ossenkop (eds.): Lusitanica et Romanica. Festschrift für Dieter Woll. Hamburgo, Helmut Verlag, págs. 293-302.

Ramajo Caño, A. (1987): Las gramáticas de la lengua castellana desde Nebrija a Correas. Salamanca, Universidad de Salamanca.

Sala, M. (1989) "La formación de palabras en el español de América. Estado actual". Homenaje a Alonso Zamora Vicente, II. Madrid, Castalia, págs. 267-278.

Serrano-Dolader, D. (1999) "Derivación verbal y parasíntesis". En I. Bosque y V. Demonte (dirs.): Gramática descriptiva de la lengua española, III. Madrid, Espasa, págs. 4683-4755.

Serrano Dolader, D. (2001): "Algunas reflexiones sobre la formación de palabras en español: notas a propósito de una publicación reciente”, Verba, 28, págs. 407-421.

Suárez Fernández, M. (1999): "La formación de palabras en algunas gramáticas del español de 1492-1771". En M. Fernández et alii (eds.): Actas del I Congreso Internacional de Historiografía Lingüística (A Coruña, 18-21 de febrero de 1997). Madrid, Arco Libros-Centro Ramón Piñeiro, págs. 627-643.

Torres Martínez, M. (2009): La prefijación en gramáticas y diccionarios del español (siglos XVIII-XX). Jaén, Universidad de Jaén [Tesis doctoral inédita].

Varela Ortega, S. y J. Martín García (1999): "La prefijación". En I. Bosque y V. Demonte (dirs.): Gramática descriptiva de la lengua española, III. Madrid, Espasa, págs. 4993-5038.

Varela Ortega, S. (2005): Morfología léxica: la formación de palabras. Madrid, Gredos. 